\section{Asymptomatic Fasciola hepatica Infection Presenting with Hypereosinophilia}

\section{Abstract}

Background: Fascioliasis is a worldwide zoonosis caused by trematodes $F$. hepatica and F. gigantica. The clinical signs, symptoms and laboratory tests are related to acute, latent or chronic phase of infection. Usually it is characterized by fever, abdominal pain, hepatosplenomegaly, high leukocyte count, vague gastrointestinal disturbances, biliary obstruction with intermittent jaundice, and eosinophilia.

Methods and Findings: A 7-year-old girl from a rural Andean area of Ecuador presented with a persistent hypereosinophilia. Leukocyte count was $35,200 \mathrm{~mm}^{3}$, with $60 \%$ eosinophilia. Anamnesis and physical examination did not reveal any signs or symptoms of infection. Ova, characteristic of Fasciola spp. were observed in the patient's stool by direct microscopic examination and confirmed to be $F$. hepática by the sequence analysis of the PCR amplicons of the second internal transcribed spacer (ITS2) gene. Abdominal ultrasonography showed a liver of normal shape, size and location with no dilation of the intra or extra-hepatic biliary tract. The patient was successfully treated with triclabendazole, $10 \mathrm{mg} / \mathrm{kg}$ for two consecutive days.

Conclusions: Clinicians and lab technicians working in endemic and non-endemic areas for fascioliasis should consider the possibility of this parasitic infection in clinically asymptomatic patients with persistent eosinophilia in order to avoid erroneous diagnosis and therapeutic interventions.

Keywords: Fascioliasis; Fasciola hepática; Andes; Ecuador

Received: January 03, 2018; Accepted: January 18, 2018; Published: January

22, 2018

\section{Introduction}

Eosinophils are white blood cells that play a vital role in fighting parasitic infections. Eosinophilia, is a term that defines blood eosinophil counts exceeding $5 \%\left(>0.5 \times 10^{9} / \mathrm{L}\right)$ of the total leucocytes count. A recent consensus on terminology defined hypereosinophilia (HE) as eosinophilia $>1.5 \times 10^{9} / \mathrm{L}$ in the peripheral blood on two occasions $>1$ month apart with or without tissue hypereosinophilia [1].

$\mathrm{HE}$ due to helminths infection has been reported with trichinellosis, strongyloidiasis, hookworm infections, ascariasis, visceral larva migrans, and fascioliasis [2,3]. F. hepática infection usually is characterized by an elevated eosinophil count, between $5 \%$ to $83 \%$, with a leukocytosis ranging from normal up to $43,000 / \mathrm{mm}^{3}[2,4-7]$. In the acute and latent-chronic phases of fascioliasis, diagnosis can be made based on eosinophilia $[8,9]$.

Fascioliasis is a zoonosis caused by trematodes $F$. hepatica and $F$.

\author{
Calvopina Manuel ${ }^{1 *}$, \\ Gonzalez Mabel'2, \\ Muñoz Greta², \\ Cevallos William³, \\ Celi Maritza ${ }^{4}$, \\ Rodriguez Richar ${ }^{4}$ and \\ Sugiyama Hiromu ${ }^{5}$
}

1. OneHealth Group, Escuela de Medicina, Facultad de Ciencias de la Salud, Universidad De Las Américas (UDLA), Quito, Ecuador

2. Servicio de Infectología, Hospital Pediátrico "Baca Ortiz" Quito-Ecuador

3. Carrera de Medicina, Facultad de Ciencias Médicas, Universidad Central del Ecuador, Quito-Ecuador.

4. Centro Internacional de Zoonosis, Universidad Central del Ecuador, QuitoEcuador.

5. Department of Parasitology, National Institute of Infectious Diseases, Tokyo, Japan

*Corresponding author:

Calvopina Manuel

manuel.calvopina@udla.edu.ec

Universidad de las Américas (UDLA), Calle Jose Queri s / n, Av. Granados and Av. Eloy Alfaro, PO BOX 17-17-9788, Quito-Ecuador.

Tel: 593-23-970000

Citation: Calvopina M, Gonzalez M, Muñoz G, Cevallos W, Celi M, et al. (2018) Asymptomatic Fasciola hepatica Infection Presenting with Hypereosinophilia. Arch Clin Microbiol. Vol.9 No.1:73

gigantica. Human infection occurs mainly due to the ingestion of uncooked aquatic vegetables containing encysted metacercariae, including watercress (Nasturtium officinale), particularly in communities where infected cattle and/or sheep are present. $F$. hepatica is widespread throughout the world, including the highlands of Bolivia, Ecuador and Peru, with children the most likely to become infected [8]. Human infections by F. gigantica 
are rare and only had been recorded in Africa and Asia continents, however in some countries like Japan both species overlap, causing identification problems [4]. In Ecuador, the incidence of human infections is quite rare, although it is highly prevalent in cattle and sheep [10]. Foodborne trematodiases, including fascioliasis, is neglected in international public health programs in comparison to other helminthic diseases [8].

The acute phase of fascioliasis is characterized by fever, abdominal pain, hepatosplenomegaly, gastrointestinal disturbances, skin rashes, high leukocyte count, eosinophilia, anemia, and elevated erythrocyte sedimentation rate (ESR); abnormal liver function tests have also been reported [4]. The latent phase is characterized by nonspecific symptoms, including vague gastrointestinal disturbances, and intermittent eosinophilia. The chronic phase is characterized by regular and constant biliary obstruction; symptoms include biliary colic pain, intermittent jaundice, eosinophilia, and right upper-quadrant abdominal tenderness. However, the percentage of asymptomatic infections is high [8].

Classical coprology (stool examination by microscopy) and serology (using enzyme-linked immunosorbent assay, ELISA) are the most commonly used diagnostic tests [8]. Neither of these methods can differentiate $F$. hepatica from $F$. gigantica. Detection of fasciolid ova in the stools of infected patients is the most definitive evidence for diagnosis. Ultrasonography and computed tomography are useful for complementary image diagnostics [11]. Molecular diagnoses by DNA isolation, real-time PCR analysis and gene sequencing have been developed with higher sensitivity, and the added benefit of species differentiation [12].

The recommended drug for treatment in all cases is triclabendazole (TCZ), $10 \mathrm{mg} / \mathrm{kg}$ of body weight, taken as a single dose. A double dose $(20 \mathrm{mg} / \mathrm{kg}$ of body weight) can be administered in case of treatment failure or if the physician judges it necessary in the management of individual cases [13].

Here, we report a parasitological and molecular confirmed human case of fascioliasis due to $F$. hepatica, presenting only with HE, and treated successfully with two doses of TCZ.

\section{Case Report}

A 7-year-old indigenous girl was referred for investigation of persistent eosinophilia which was found incidentally during a routine health control at her primary school. She was born and resides in a community of the Chimborazo province. It is located in the Andean mountains range of central Ecuador, at an altitude of $3200 \mathrm{~m}$, approximately $310 \mathrm{~km}$ from the capital of Quito. This is a temperate region with temperatures ranging from $5^{\circ} \mathrm{C}$ to $12^{\circ} \mathrm{C}$. The area, characterized by the Andean high plateau ecosystem, is fragmented into small parcels for cattle ranching, with scattered houses mainly inhabited by Kichwa indigenous people. Cows, sheep and pigs are the animals raised in this region.

The girl was admitted to the Children Hospital in Quito, with a 5-months history of persistent hypereosinophila. Past medical history revealed her not having any allergies or taking any medications. Eosinophilia of $42 \%$ of the total leucocyte count was detected for the first time (July 2014) during a routine health control for school children. The second and third time, eosinophilia was $60 \%$ and $44 \%$, respectively. On admission (December 2014), the patient was non-febrile, with no abdominal pain or diarrhea. According to her mother, she has a history of anorexia. Upon questioning of her food consumption and eating habits, her mother admitted of the family eating watercress (Nasturtium officinale) prepared in salads, either grown in their backyard or collected elsewhere in the community. The family raises sheep, cows and chickens. In the community, animals are allowed to roam freely throughout the area.

During the physical exam, the vital signs were all within the normal limits for her age; her axillar temperature was $36.7^{\circ} \mathrm{C}$, the respiratory rate was 20 breaths/min, and her heart rate was 88 beats $/ \mathrm{min}$. She was at the 3 th percentile for weight $(17.4 \mathrm{~kg})$ and $>3^{\text {th }}$ percentile for height $(110 \mathrm{~cm})$. There was no pain upon abdominal palpation. No hepatosplenomegaly, ascites, or masses were found. There was no evidence of lymphadenopathy or skin rash. The rest of the physical examination was unremarkable. Blood tests showed a white blood cell count of $17.1 \times 10^{9} / L$, with $8 \%$ neutrophils, $28 \%$ lymphocytes, and $64 \%$ eosinophils. The ESR was $18 \mathrm{~mm} / \mathrm{h}$, with normal hemoglobin $(13.1 \mathrm{~g} / \mathrm{dL})$, a hematocrit of $38.4 \%$, and a platelet count of $571,000 / \mu \mathrm{L}$. Serology, using Fasciola excretory-secretory antigens (FES-Ag) for detection of anti-Fasciola antibodies in serum, was positive with a titration of $1 / 2560$. All results of the basic metabolic panel were within normal limits. Aspartate aminotranferase (AST) level was at 41 $\mathrm{IU}$, serum alanine transferase (ALT) at $44 \mathrm{IU}$, gama glutamyl transferase at $50 \mathrm{U} / \mathrm{L}$, lactodehydrogenase at $410 \mathrm{U} / \mathrm{L}$, and procalcitonine at $0.09 \mathrm{ng} / \mathrm{mL}$; all within normal limits for her age.

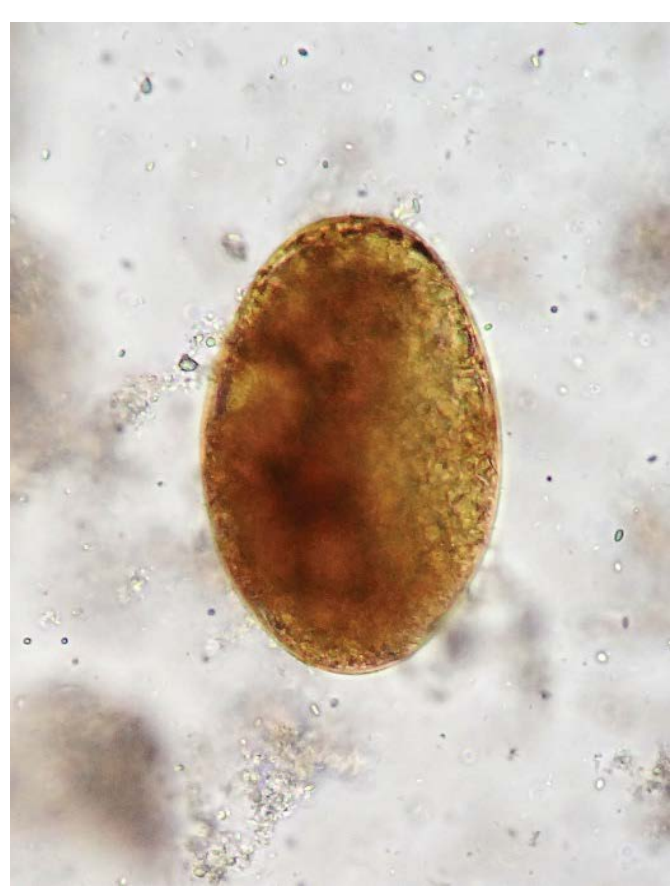

Figure 1 An ovoid, yellow-green, operculated ova observed in the stool of the 7-year-old girl. The ova measured $137 \times 70$ um. (400x magnification). 
Urine test results were normal. Stool examination performed for three consecutive days showed numerous operculated ova, yellow-green in color, and measuring approximately $138 \times 70 \mu \mathrm{m}$, characteristic of $F$. hepatica (Figure 1). All stool specimens were negative for any other intestinal parasites. The ultrasound image revealed a liver with normal shape, size and location. There was heterogeneous echogenicity especially in the left lobe. No dilation of intra or extra-hepatic biliary tract was found. The gallbladder was thin walled and internally showed a motile hyperechogenic image with irregular borders.

Molecular characterization was performed by DNA isolation from eggs, and subsequent amplification of the ITS2 region of the ribosomal RNA gene by PCR as described previously [14]. The primers used for amplification and sequencing were $3 \mathrm{~S}$ (forward, 5'-GGTACCGGTGGATCACTCGGCTCGTG-3') [15]; and A28 (reverse, 5'-GGGATCCTGGTTAGTTTCTTTTCCTCCGC-3') [15]. The amplified products were sequenced using the corresponding primers and the BigDye Terminator v3.1 Cycle Sequencing Kit (Thermo Fisher Scientific) on an automated sequencer (3730xl DNA Analyzer, Thermo Fisher Scientific). Sequences were aligned and compared using GENETYX-Win software (Ver. 13, Genetyx Co., Tokyo, Japan). ITS2 products of about 590 base pairs (bp) were generated. The sequence analysis of the PCR amplicons revealed that the amplified product was $538 \mathrm{bp}$ (without primer sequences). Nucleotide sequence has been deposited as the ova stage of $F$. hepatica in the DDBJ/EMBL/GenBank database, accession number L0056930.

TCZ was used for treatment as recommended by the guidelines of WHO (2011), $10 \mathrm{mg} / \mathrm{kg} /$ day for 2 days. The medication was well-tolerated. The patient was evaluated at $1,3,6$, and 12 months post-treatment and all stool examinations were negative for Fasciola spp. ova. Her mother signed a consent to participate in this publication.

\section{Discussion}

The present report highlights that in asymptomatic patients, who present with only hypereosinophilia (HE), fascioliasis must be considered in the differential diagnosis, particularly when patients came from rural Andean regions. Furthermore, the history of ingestion of aquatic plants like watercress in salads and raising of cows, sheep and pigs indicate potential risks of acquiring $F$. hepatica infection. On the other hand, the DNA isolated from eggs analyzed molecularly identified them as F. hepatica and the sequence obtained was identical to those established from adult flukes of $F$. hepatica occurring in cattle in the same Andes region of Ecuador (DDBJ/EMBL/GenBank database, accession number LO056929).

Eosinophilia is not an uncommon finding in clinical practice

\section{References}

1 Datt LA, Varma S (2015) Management of hypereosinophilia in tropical settings. Medical Journal Armed Forces India 71: 60-66.

2 Cihan MK, Babur C, Olcay L (2013) Infection as a Cause of Severe Hypereosinophilia. Turk J Hematol 30: 88-90. and, when present and used in conjunction with signs and symptoms, can serve as a useful clue in the differential diagnosis. Contrarily, the etiology of only eosinophilia can be difficult to identify in asymptomatic patients [17]. With a Fasciola spp. infection, usually during acute and chronic phases, eosinophilia with symptoms and signs are present, but the latent phase is characterized by nonspecific symptoms [4]. In the present case, clinical symptoms and signs were not present. In contrast, leukocytosis with HE was persistent for 5 months, indicating this case was probably in the latent phase. Elevated levels of immunoglobulins against $F$. hepatica were present as detected by the ELISA method, supporting that this case was not in the acute phase. In addition, the ultrasound confirmed the patient did not have hepatomegaly. In the chronic phase, the majority of infected patients have anemia, elevated ESR, and abnormal liver function tests [4] all of which were negative in our case. The present patient corroborated with the WHO (2007) statement that the percentage of asymptomatic fascioliasis cases is apparently high [9].

In Ecuador, human infection by $F$. hepatica has rarely been diagnosed and reported, although Ecuador has been considered highly endemic for Fasciola infection [8]. Since the first recognized case in the year 1969 [18] only 33 cases have been documented by eggs observation, 9 cases using ELISA test for FES-antigens $[10,19]$ and 4 cases by the FasciDIG method [20]. All were from the Andean highland communities, as is the present case. Notwithstanding, veterinary fascioliasis is quite common with prevalence of 10 to $60 \%$ in cattle from Andean localities [20]. This information should alert both physicians and researchers of the possibility that human fascioliasis can also be found in all Andean plateaus. An active search for human infection in Ecuadorian Andean communities has revealed a prevalence of infection from $1.7 \%$ to $6 \%[10,20]$ indicating the importance of performing further community-based or seroepidemiological surveys to determine the real and actual prevalence of infection.

TCZ administered postprandial at $10 \mathrm{mg} / \mathrm{kg}$ body weight for 2 consecutive days, as recommended [13] was effective in curing this case. No adverse reactions to the medication was noted. TCZ is not listed in the Ecuadorian National Guidelines for Basic Drugs [21] and is not available in the country. Health authorities have not requested TCZ from WHO, which will donate it upon request.

In conclusion, it is imperative that clinicians and lab technicians working in endemic and non-endemic areas for fascioliasis consider the possibility of this parasitic infection in clinically asymptomatic patients with persistent eosinophilia in order to avoid erroneous diagnosis and therapeutic interventions. Patient management also requires knowledge concerning the usage of both the diagnostic tests and the specific drugs needed to treat the infection.

3 Ecevit C, Bağ O, Vergin C, Öztürk A (2013) Visceral Larva Migrans Presenting with Hypereosinophilia. Turkiye Parazitol Derg 37: 58-60.

4 Mas-Coma S, Bargues MD (1997) Human liver flukes: A review. Research and reviews in Parasitology 57: 145-218.

5 Necati HI, Aktaş G, Savli H, Küçükbayrak A, Gürel S (2012) A Fascioliasis 
Case: a not Rare Cause of Hypereosinophilia in Developing Countries, Present in Developed too. Mediterr J Hematol Infect Dis 4: 1-4.

6 Gökçe M, Şahiner Ü, Ünal Ş, Parlakay A, Önce I, et al. (2011) An unexpected parasitic cause of hypereosinophilia: fascioliasis. Turkish J Pediat 53: 111-113.

7 Noyer CM, Coyle CM, Werner C, Dupouy-Camet J, Tanowitz HB, et al. (2002) Hypereosinophilia and liver mass in an immigrant. Am J Trop Med Hyg 66: 774-776.

8 WHO (2007) Report of the WHO Informal Meeting on use of triclabendazole in fascioliasis control. WHO headquarters, Geneva, Switzerland 22: 154.

9 Adachi S, Kotani K, Shimizu T, Tanaka K, Shimizu T, et al. (2005) Asymptomatic Fascioliasis. Internal Medicine 449: 1013-1015.

10 Trueba G, Guerrero T, Fornasini M, Casariego I, Zapata S, et al. (2000) Detection of infection in a community located in the Ecuadorian Andes. Am J Trop Med Hyg 62: 518.

11 Van Beers B, Pringot J, Geubel A, Trigauxc JP, Bigaignon G, et al. (1990) Hepatobiliary fascioliasis: Noninvasive Imaging Findings. Radiology 174: 809-810.

12 Calvani NED, Windsor PA, Bush RD, Šlapeta J (2017) Scrambled eggs: A highly sensitive molecular diagnostic workflow for species specific detection from faecal samples. PLoS Negl Trop Dis 11: 1-18.

13 WHO (2011) Report of the WHO Expert Consultation on Foodborne
Trematode Infections and Taeniasis/Cysticercosis. Vientiane, Lao People's Democratic Republic, WHO/HTM/NTD/PCT/2011 3: 12-16.

14 Sugiyama H, Singh TS, Rangsiruji A, Paragonimus R (2013) In Liu DY ed. Detection of Human Parasitic Pathogens. Boca Raton, Florida, USA. CRC Press 23: 423-436.

15 Bowles J, Blair D, McManus DP (1999) A molecular phylogeny of the human schistosomes. Mol Phylog Evol 4: 103-109.

16 Blair D, Agatsuma T, Watanobe T, Okamoto M, Ito A (1997) Geographical genetic structure within the human lung fluke, detected from DNA sequences. Parasitology 115: 411-417.

17 Chen Yun-Yun K, Khoury P, Ware JM, Holland-Thomas NC, Stoddard $\mathrm{JL}$, et al. (2014) Marked and persistent eosinophilia in the absence of clinical manifestations. J Allergy Clin Immunol 133: 1195-1202.

18 Ontaneda M, Ribadeneira J, Chavez J (1968) Fascioliasis hepática. Presentacion de un caso. Rev Ecuat Medic y Cienc Biol 6: 87-93.

19 Leon LA, Leon R (1980) Las parasitosis hepatobiliares en el Ecuador. Rev Ecuat Med XVI 1: 7-18.

20 Narváez A (1989) Prevalencia y factores asociados a la otras parasitosis intestinales en la comunidad de Tarqui, Ecuador. Tesis de Maestria. Cuidad de la Habana 22: 121.

21 CNMB (2014) (Cuadro Nacional de Medicamentos Básicos). Ministerio de Salud Pública del Ecuador. Publiasesores Cia.Ltda, Quito 15: 1-894. 